\title{
Betriebliche Gesundheitsförderung in Deutschland für alle? Ergebnisse der BIBB-|BAuA-Erwerbstätigenbefragung 2018
} Workplace Health Promotion in Germany for All? Results of the 2018 BIBB/BAuA survey of Employed People

\section{(c) (1) $(8) \odot$}

\author{
Autor \\ Alfons Hollederer
}

Institut

Fachbereich 01 Humanwissenschaften, Universität Kassel, Kassel, Deutschland

\section{Schlüsselwörter}

Gesundheitsförderung, Betriebliche Gesundheit, Gesundheitszustand, Prävention, Arbeitszufriedenheit, Arbeitsplatz

Key words

Health promotion, Occupational health, Health Status,

Prevention, Job Satisfaction, Workplace

online publiziert $\quad 10.11 .2021$

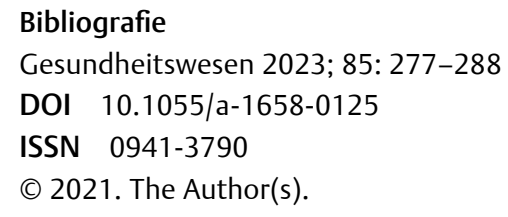

This is an open access article published by Thieme under the terms of the Creative Commons Attribution-NonDerivative-NonCommercial-License, permitting copying and reproduction so long as the original work is given appropriate credit. Contents may not be used for commercial purposes, or adapted, remixed, transformed or built upon. (https://creativecommons.org/ licenses/by-nc-nd/4.0/)

Georg Thieme Verlag, Rüdigerstraße 14,

70469 Stuttgart, Germany

\section{Korrespondenzadresse}

Prof. Dr. Alfons Hollederer

Universität Kassel

Fachbereich 01 Humanwissenschaften

Arnold-Bode-Straße 10

34127 Kassel

Deutschland

alfons.hollederer@uni-kassel.de

\section{ZUSAMMENFASSUNG}

Ziel der Studie Die Sekundäranalyse zielt darauf, die Verbreitung und Inanspruchnahme der Betrieblichen Gesundheitsförderung (BGF) in Deutschland zu untersuchen.

Methodik Datenbasis ist die BIBB-/BAuA-Erwerbstätigenbefragung 2018. Sie ist eine randomisierte repräsentative CATIErhebung von 20.012 Erwerbstätigen ab 15 Jahren in Deutsch- land. Es werden Anteilswerte zwischen verschiedenen Gruppen verglichen und eine binäre logistische Regressionsanalyse durchgeführt.

Ergebnisse $47 \%$ der Erwerbstätigen berichten, dass in ihrem Betrieb in den letzten 2 Jahren Maßnahmen der BGF durchgeführt wurden. 25\% der Erwerbstätigen haben an einem Angebot zur BGF in den letzten 2 Jahren teilgenommen. Mit der Betriebsgröße nehmen die Anteile der Erwerbstätigen, die ein BGF-Angebot im Betrieb bestätigen, stark zu, aber die Inanspruchnahmequoten sinken. Die Erwerbstätigen in Betrieben mit Betriebs-/Personalrat geben überproportional häufig eine Durchführung der BGF an. Erwerbstätige mit niedrigem sozio-ökonomischen Status bekunden dagegen in relativ geringem Ausmaß BGF-Angebote. BGF wird besonders wenig in Betrieben von Erwerbstätigen ohne Berufsabschluss, in einem befristeten Arbeitsverhältnis, mit Entlassungsgefahr und Leiharbeitnehmern umgesetzt. Binäre logistische Regressionsanalysen zielen darauf, Prädiktoren des BGF-Angebots zu identifizieren. Im Vergleich zu den Kleinbetrieben ist bei den Erwerbstätigen in mittleren Unternehmen die Wahrscheinlichkeit für ein BGF-Angebot in ihrem Betrieb mehr als doppelt $(O R=2,17)$ und in Großunternehmen mehr als fünffach erhöht $(O R=5,64)$. Im Öffentlichen Dienst stehen die Chancen von Erwerbstätigen auf BGF im Betrieb wesentlich besser als in Industrie, Handwerk, Handel oder weiteren Bereichen. Erwerbstätige mit ausländischer Staatsangehörigkeit haben im Vergleich zu deutschen Erwerbstätigen eine signifikant geringere Chance, dass in ihrem Betrieb BGF angeboten wird $(O R=0,78)$. Ein sehr guter oder ausgezeichneter Gesundheitszustand geht mit einer etwas gesteigerten OR von 1,15 für BGF im Betrieb einher. In Betrieben, in denen die Erwerbstätigen mit der Arbeit insgesamt sehr zufrieden sind, ist die Wahrscheinlichkeit eines BGF-Angebots deutlich erhöht $(O R=1,70)$.

Schlussfolgerung Die Studie zeigt große Disparitäten in der BGF auf. Es braucht zielgruppenspezifische Handlungsstrategien in der BGF, um sowohl Kleinbetriebe als auch innerhalb von Betrieben die Erwerbstätigen mit niedrigem sozio-ökonomischen Status besser zu erreichen.

\section{ABSTRACT}

Aim of the study This secondary analysis aims to investigate the prevalence and uptake of workplace health promotion (WHP) in Germany. 
Methods Our data base was the 2018 BIBB-/BAuA Survey in Germany, which is a randomized representative CATI survey of 20,012 employed persons aged 15 and older. Proportions were compared among different groups and a binary logistic regression model was estimated.

Results $47 \%$ of the employees reported that WHP interventions had been implemented in their company in the last two years. $25 \%$ of the employees had taken up the offer of WHP. The proportion of employees who confirmed that WHP was offered in their company increased sharply with the company size, but the take-up rates decreased. Employees in companies with a works/staff council stated a disproportionately high rate of implementation of WHP. However, employees with a low socioeconomic status reported WHP offer to a relatively small extent. In particular, employees without a vocational qualification, in a fixed-term employment, at risk of dismissal and temporary agency workers reported a relatively low implementation of WHP in their company. Logistic regression analyses aimed to identify predictors for the WHP offer. Compared to small companies, workers in medium-sized companies were more than twice as likely $(\mathrm{OR}=2.17)$ to have a WHP offer in their company and more than five times as likely $(O R=5.64)$ in large companies. In the public sector, the chances of employees having WHP in their company were significantly better than in industry, crafts, trade or other sectors. Compared to German employees, employees with a foreign citizenship had a significantly lower chance of WHP being offered in their company $(\mathrm{OR}=0.78)$. A very good or excellent health status was associated with a slightly increased OR of 1.15 for WHP in the company. In companies in which employees were very satisfied with their work overall, the probability of a WHP offer was significantly increased $(O R=1.70)$. Conclusion The analysis reveals large disparities in the WHP. There is a need for WHP strategies for targeting the small companies and the employees with a low socioeconomic status within the companies.

\section{Einleitung}

„Wir bieten Ihnen ein Betriebliches Gesundheitsmanagement mit zahlreichen Angeboten zur Gesundheitsförderung “ verheißen Stellenausschreibungen potenziellen Bewerbern als komparativen Einstellungsvorteil, wie in diesem Beispiel aus der Stellenbörse INTERAMT. Die Bedeutung der betrieblichen Gesundheitsförderung (BGF) hat in Deutschland mit dem Fachkräftemangel, dem demografischen Wandel und der Erhöhung der Lebensarbeitszeit weiter zugenommen. Die BGF folgt dem in der Ottawa-Charta der WHO angelegten Setting-Ansatz der gesundheitsförderlichen Lebenswelten: „Die Art und Weise, wie eine Gesellschaft die Arbeit, die Arbeitsbedingungen und die Freizeit organisiert, sollte eine Quelle der Gesundheit und nicht der Krankheit sein. " [1] Die BGF umfasst nach der Luxemburger Deklaration des Europäischen Netzwerks für Betriebliche Gesundheitsförderung „alle gemeinsamen Maßnahmen von Arbeitgebern, Arbeitnehmern und Gesellschaft zur Verbesserung von Gesundheit und Wohlbefinden am Arbeitsplatz.“ [2] Die Handlungsansätze sind dabei in der Praxis vielfältig. Die Luxemburger Deklaration [2] benennt die Verbesserung der Arbeitsorganisation und der Arbeitsbedingungen, die Förderung einer aktiven Mitarbeiterbeteiligung und die Stärkung persönlicher Kompetenzen. In Abgrenzung zum Arbeits- und Gesundheitsschutz geht es bei der BGF um freiwillige Aktivitäten von Arbeitgebern und Erwerbstätigen.

Die Studienlage zur Verbreitung und Inanspruchnahme der BGF hat sich in Deutschland verbessert, ergibt aber in der Gesamtschau ein heterogenes Bild [3]. Die wichtigsten Datenquellen für den Verbreitungsgrad der BGF in Deutschland stellen das IAB-Betriebspanel mit den Wellen 2002, 2004 und 2012 [4, 5] und die BIBB/BAuAErwerbstätigenbefragung mit den Erhebungswellen 2005/2006 und 2011/12 dar [6]. Trotz der verschiedenen Erhebungsmethoden, einerseits über Arbeitgeber und andererseits über Erwerbstätige, kommen beide Quellen zu einer sehr hohen Übereinstimmung in den Hauptaussagen und belegen einen moderaten Ausbau der BGF in Deutschland in den letzten beiden Dekaden. Nach dem IAB Betriebspanel stieg der Anteil der Betriebe mit BGF von 19\% im Jahr
2002 auf $20 \%$ im Jahr 2004 und wuchs schließlich auf $27 \%$ im Jahr 2012 an [4, 5]. Als Markenzeichen der BGF gilt der Gesundheitszirkel [7], der aber im IAB Betriebspanel 2012 nur bei $6 \%$ der Betriebe durchgeführt wurde. Es dominierten Mitarbeiterbefragungen und Arbeitsunfähigkeitsanalysen vor Präventionskursen und Gesundheitsförderungsmaßnahmen. In der repräsentativen BIBB/BAuAErwerbstätigenbefragung nahm gemäß einer Auswertung von Beck und Lenhardt [6] der Anteil der Erwerbstätigen, die Maßnahmen der BGF in ihrem Betrieb berichteten, von 38 auf $44 \%$ zwischen Welle 2005/2006 und 2011/12 zu. Nach einem indirekten Schätzmodell kommen sie für die Erhebungswelle 2011/12 auf eine Beteiligung von $26 \%$ der Betriebe, was dem Verbreitungsgrad von BGF im IAB Betriebspanel 2012 entspricht [6]. Insgesamt engagiert sich also nur eine deutliche Minderheit der Betriebe, in der aber ein Großteil der Beschäftigten arbeitet, weil die Umsetzung der BGF stark mit der Betriebsgröße ansteigt.

Darüber hinaus gibt es eine Reihe von empirischen Studien in Deutschland, die sich aber in der Sensitivität der Messung der BGF und im Beobachtungszeitraum erheblich unterscheiden [8-10] oder sich selektiv auf einzelne Regionen, Branchen, Betriebsgrößen und Altersgruppen konzentrieren [11-19]. Die Grenzen zwischen BGF und dem umfassenderen Betrieblichen Gesundheitsmanagement werden mit der Komplexität der Programme zunehmend fließend und führen je nach Erhebungskonzeption zu verschiedenen Prävalenzraten der BGF [3] und Maßnahmeninhalten. Im Gesundheitssurvey GEDA 2014/2015-EHIS gaben beispielsweise knapp ein Drittel der erwerbstätigen Männer und fast ein Viertel der erwerbstätigen Frauen an, dass es in ihrem Betrieb Angebote zur Rückengesundheit im letzten Jahr gab.

Gemäß § 20b SGB V können Krankenkassen den Aufbau und die Stärkung gesundheitsförderlicher Strukturen in den Betrieben unterstützen und Präventionsmaßnahmen (wie Stressbewältigung, Bewegungsförderung, gesunde Ernährung und Suchtprävention) fördern. Im Jahr 2019 erreichte die gesetzliche Krankenversicherung (GKV) direkt 23221 Betriebe mit schätzungsweise über 2 Mio. Beschäftigten und verausgabte dafür rund 240 Mio. Euro [20]. Die 
private Kranken- und Pflegeversicherung (PKV), die auch den Großteil der Beamtenschaft versichert, ist nicht in der BGF engagiert [21].

Da arbeitsbedingte Gesundheitsrisiken sozial ungleich verteilt sind, kommen gesundheitsbezogenen Interventionen in der $\mathrm{Ar}$ beitswelt wie die BGF besondere Wichtigkeit zu [22]. Auch in Deutschland ist eine höhere Prävalenz von Arbeitsbelastungen bei manuellen Berufen und einfachen Angestellten [23] sowie bei niedriger Qualifikation [24] nachgewiesen. Wichtige Hinweise auf gesundheitliche Ungleichheit in der Arbeitswelt geben auch die Arbeitsunfähigkeitsanalysen der Krankenkassen, da bspw. die Krankenstände bei niedrigem oder keinem Berufsabschluss stark erhöht sind [25]. Arbeitsunfähigkeitsanalysen werden häufig für die Bedarfsermittlung der BGF herangezogen. Der § 20 SGB V Abs. 1 sieht für die GKV auch vor, dass Leistungen zur primären Prävention und Gesundheitsförderung ,insbesondere zur Verminderung sozial bedingter sowie geschlechtsbezogener Ungleichheit von Gesundheitschancen beitragen [sollen]“. Allerdings wird der Sozialstatus der Maßnahmeteilnehmer in der Präventionsberichterstattung der GKV und der Sozialleistungsträger der Nationalen Präventionskonferenz nicht erfasst [20,21]. Die Bundesregierung regte deshalb an, die fehlenden Kennzahlen zur Verminderung sozial bedingter und geschlechtsbezogener Ungleichheit von Gesundheitschancen einzuführen [26].

Die Effektivität und Effizienz der BGF kann alles in allem als wissenschaftlich abgesichert gelten. Der internationale Forschungsstand zeigt, dass die BGF die Gesundheit verbessert, zur Reduktion von Krankenständen führt und die Arbeitsfähigkeit erhöht [27-31]. Die Effektgrößen sind eher moderat. BGF kann auch Veränderungen des Gesundheitsverhaltens in kleinem Ausmaß erreichen $[28,31]$. Ein Return-on-Investment ist für die BGF nach der Studienlage durch die erreichten Verbesserungen positiv [28, 32].

Ziel der vorliegenden Sekundäranalyse ist es, die aktuelle Verbreitung und Inanspruchnahme der BGF in Deutschland zu untersuchen und Prädiktoren des BGF-Angebots zu identifizieren. Sie zielt auch darauf, das Forschungsdefizit über den Erreichungsgrad von sozial benachteiligten Erwerbstätigengruppen durch die BGF zu schließen.

\section{Methode}

Für diese Sekundäranalyse werden Daten der „BIBB/BAuA-Erwerbstätigenbefragung 2018 - Arbeit und Beruf im Wandel. Erwerb und Verwertung beruflicher Qualifikationen“ genutzt. Sie wurden vom Bundesinstitut für Berufsbildung (BIBB) und der Bundesanstalt für Arbeitsschutz und Arbeitsmedizin (BAuA) von Oktober 2017 bis April 2018 mittels computergestützter telefonischer Interviews (CATI) erhoben [33]. Der Datenzugang erfolgte über einen Scientific-Use-File des Forschungsdatenzentrums im BIBB (BIBB-FDZ). Die Grundgesamtheit der Repräsentativerhebung bilden Kernerwerbstätige in Deutschland, die mindestens 15 Jahre alt sind und einer bezahlten Arbeit von mindestens 10 Stunden pro Woche nachgehen. Die Zufallsstichprobe umfasst 20012 Erwerbstätige. Für die Hochrechnung enthält der Scientific-Use-File ein Anpassungsgewicht (Variablen Ost/West, BIK, Haushaltsgröße, Stellung im Beruf, Geschlecht, Nationalität, Bildung, Alter). Die Referenzstrukturen liefert der Mikrozensus 2017. Die Erhebungsmethode ist ausführlich bei Rohrbach-Schmidt und Hall [34] und die Stich- probe bei Lück et al. [33] beschrieben. Im Fokus der vorliegenden Auswertung steht die BGF, die in der BIBB/BAuA-Erwerbstätigenbefragung 2018 wie folgt erfragt wurde:

1) Wurden in Ihrem Betrieb in den letzten 2 Jahren Maßnahmen der Gesundheitsförderung durchgeführt? (Ja/Nein/Weiß nicht) 2) Haben Sie daran teilgenommen? (Ja/Nein).

In der Befragung wurden bei den Erwerbstätigen die Zahl der Beschäftigten und die berufliche Stellung in ihrer Tätigkeit erfasst (mit Antwortoptionen Arbeiter, Angestellte, Beamte, Selbstständige, freiberuflich Tätige, freie Mitarbeiter, mithelfende Familienangehörige). In der Filterführung der CATI-Erhebung wurden aus Plausibilitätsgründen den Selbstständigen und freiberuflich Tätigen in Ein-Personen-Betrieben die Fragen zur BGF nicht vorgelegt. Die BIBB/BAuA-Erhebung beinhaltet Fragen nach den Tätigkeiten der Erwerbstätigen, ihren Arbeitsbedingungen und der Arbeitszufriedenheit. In der Analyse werden Assoziationen zwischen der BGF und folgenden Items untersucht:

- Merkmale des Betriebs (Beschäftigtenzahl, Betriebs-/ Personalrat, Wirtschaftszweige WZ 2008 [35], Leiharbeit)

- sozio-demografische Variablen (Geschlecht, Alter, höchster Ausbildungsabschluss, Staatsangehörigkeit, Stellung im Beruf)

- amtlich anerkannte Behinderung und subjektiver Gesundheitszustand,

- arbeitsplatzbezogene Merkmale (Befristung, Entlassungsgefahr, Arbeitszufriedenheit),

- Klassifikation der European Socioeconomic Groups [ESeG]. Sie ist ein Instrument zur Messung des sozioökonomischen Status, das auf Basis der internationalen Standardklassifikation der Berufe (ISCO-082) und des Beschäftigungsstatus (Anstellung/Selbstständigkeit) gebildet wird [36, 37].

In der Methodik verwendet der Untersuchungsansatz deskriptive Statistik, Korrelationsanalytik und binäre logistische Regressionsanalysen. Für die Testung von Unterschiedshypothesen werden ChiQuadrat-Tests nach Pearson durchgeführt. Als Zusammenhangsmaße für nominalskalierte Variablen werden bei alternativen Variablen die Phi-Koeffizienten genutzt. Die Odds Ratio beziehungsweise das Chancenverhältnis ist in den logistischen Regressionsanalysen ein Maß dafür, um wie viel größer die Wahrscheinlichkeit eines Ereignisses (wie Angebot der BGF) in einer Gruppe mit bestimmten Merkmalen im Vergleich zur Gruppe ohne diese Merkmale ist. Zu den Odds Ratios werden 95\%-Konfidenzintervalle berechnet. Die Modellvoraussetzungen werden überprüft. Die Analysen wurden mit IBM/SPSSStatistics 26 durchgeführt.

\section{Ergebnisse}

- Tab. 1 gibt eine Übersicht über die Stichprobe in der BIBB-/BAuAErwerbstätigenbefragung 2018 mit Grundauszählungen und informiert über Häufigkeitsverteilungen bei der Durchführung und Teilnahme der BGF. 47\% der interviewten Erwerbstätigen berichten, dass BGF in den letzten 2 Jahren in ihrem Betrieb angeboten wurde. Von ihnen haben 59\% BGF-Maßnahmen auch in Anspruch genommen.

- Tab. 1 zeigt bedeutsame Disparitäten bei Angebot und Inanspruchnahme der BGF zwischen den Gruppen nach sozio-demo- 


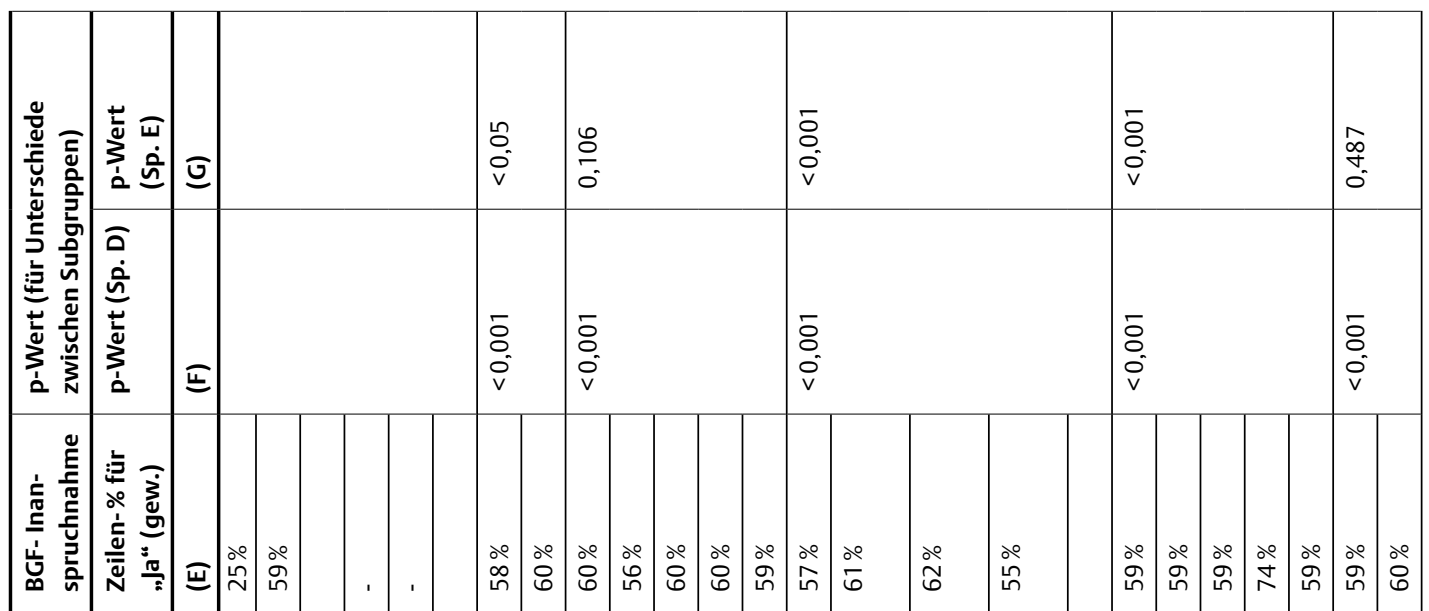

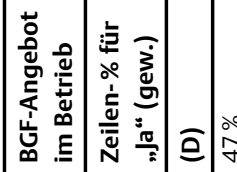

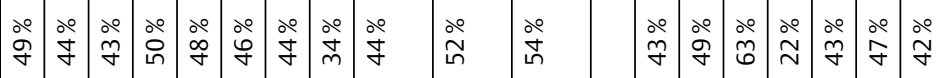
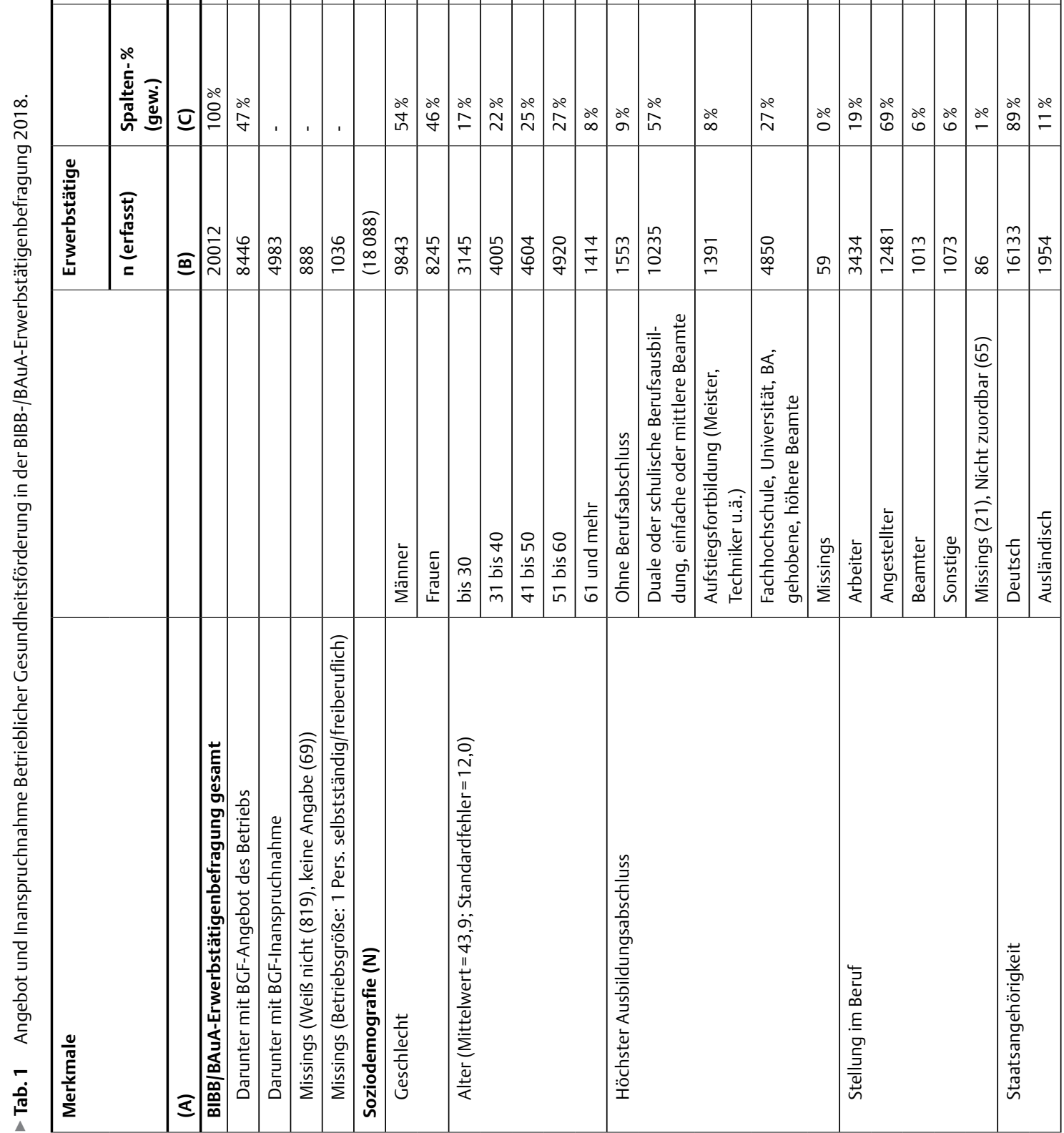


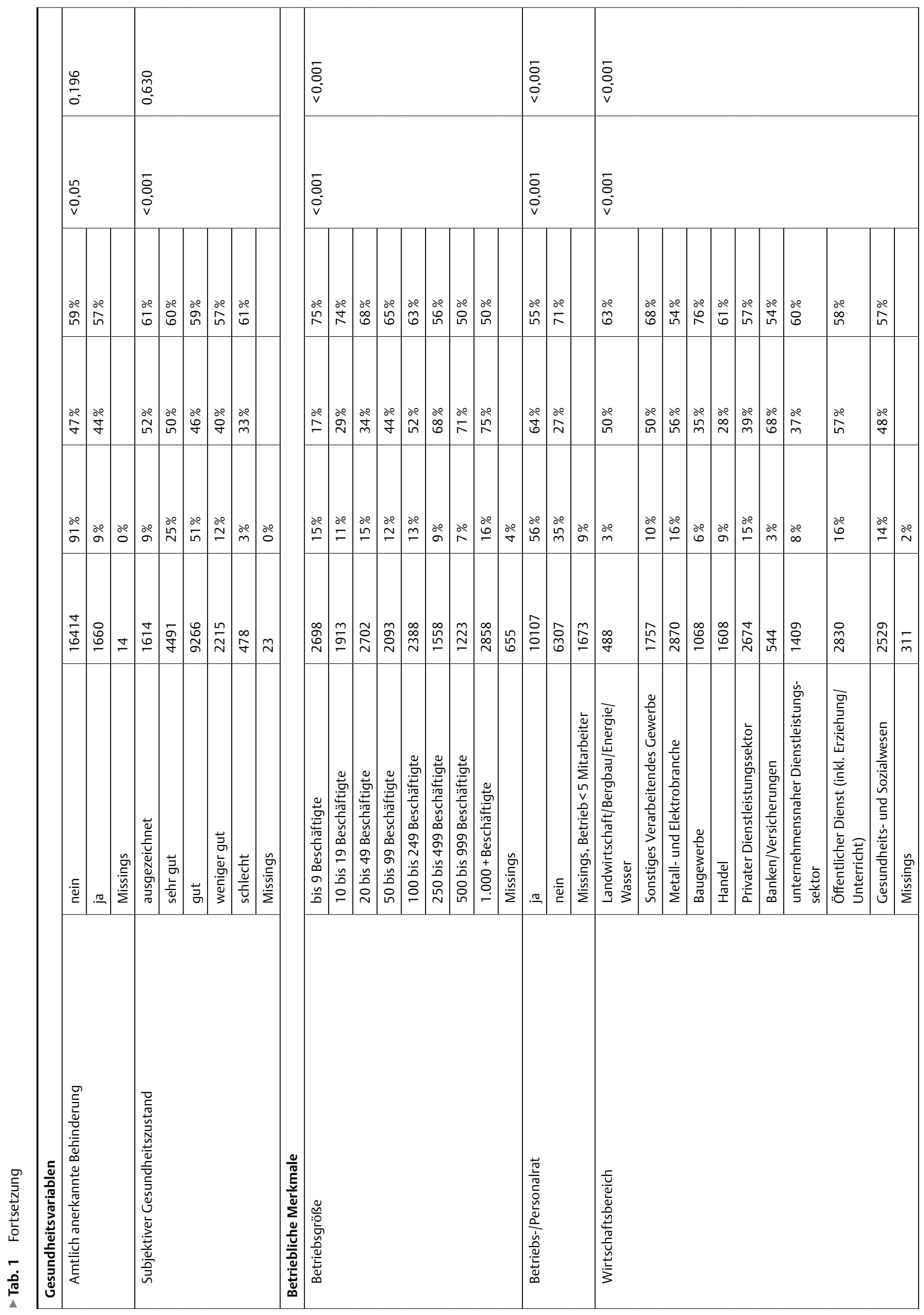




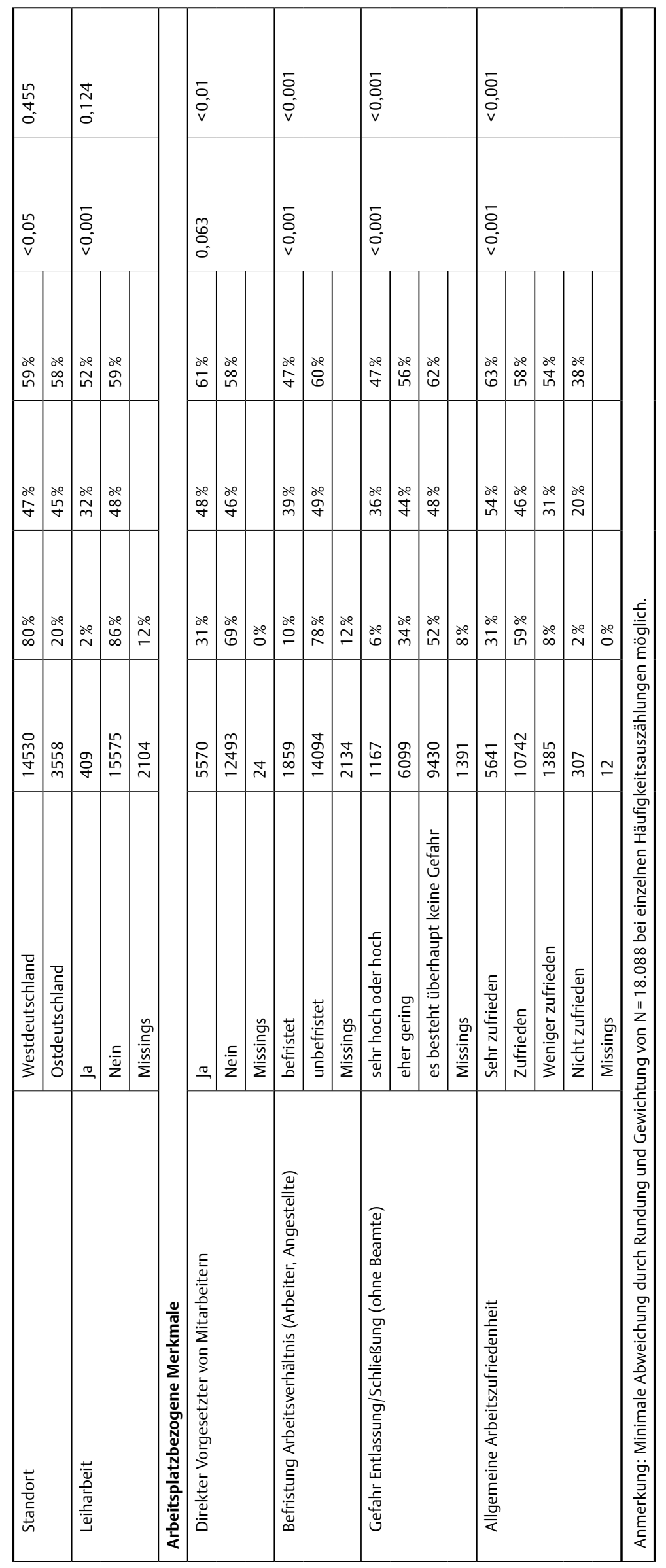


grafischen Merkmalen und arbeitsweltbezogenen Variablen auf. Erwerbstätige im mittleren Lebensalter geben im Verhältnis häufiger die Durchführung von BGF im Betrieb als die anderen Altersgruppen an. Männer berichten etwas mehr ein BGF-Angebot als Frauen, die es dann wiederum in leicht höherem Maße wahrnehmen.

Je höher der Ausbildungsabschluss der Erwerbstätigen, desto häufiger wird die Umsetzung der BGF im Betrieb genannt. Während lediglich bei $34 \%$ der Erwerbstätigen ohne Berufsabschluss BGF im Betrieb angeboten wurde, liegt der korrespondierende Anteil bei Erwerbstätigen mit Hochschulabschluss bei $54 \%$.

In den Dienststellen von Beamten gibt es wesentlich häufiger ein BGF-Angebot (63\%) als bei Arbeitern (43\%), Angestellten (49\%) und sonstigen Erwerbstätigen. Bei Letzteren ist der Anteil mit BGFAngeboten besonders gering, die Inanspruchnahme dagegen auffallend hoch (22 und 74\%).

Bei den Erwerbstätigen mit amtlich anerkannten Behinderungen (44\%) wird etwas weniger häufig als bei den übrigen Erwerbstätigen (47\%) BGF im Betrieb durchgeführt. Wie \ Tab. 1 offenlegt, sind BGF-Angebote mit dem selbstbewerteten Gesundheitszustand der Erwerbstätigen assoziiert. Je schlechter der subjektive Gesundheitszustand, desto weniger werden BGF-Angebote bekundet.

Mit der Betriebsgröße nehmen die Durchführung der BGF massiv zu und die Inanspruchnahmequoten jedoch stark ab. In Betrieben bis zu 9 Beschäftigten geben nur 17\% der Erwerbstätigen an, dass BGF angeboten wurde. Davon haben drei Viertel der Erwerbstätigen teilgenommen. In Betrieben mit mindestens 1000 Beschäftigten liegt der Anteil der Erwerbstätigen mit BGF-Angebot dagegen bei $75 \%$, von denen die Hälfte annahm.

Angebote und Inanspruchnahme der BGF korrelieren stark mit dem Vorhandensein eines Betriebs-/Personalrats (in den Betrieben mit mindestens 5 Beschäftigten). Wie die $>$ Abb. 1 verdeutlicht, ist in allen untersuchten Betriebsgrößenklassen ein signifikant höherer Anteil von Erwerbstätigen mit BGF-Angeboten in Betrieben mit
Betriebs-/Personalrat zu beobachten. Der Zusammenhang ist besonders groß in Kleinbetrieben, in denen aber bekanntermaßen selten Betriebs-/Personalräte gegründet werden.

Die Verbreitung der BGF variiert stark nach Wirtschaftsbereichen ( $\triangleright$ Tab. 1) bzw. Wirtschaftszweigen. Am wenigsten berichten Erwerbstätige BGF-Angebote im Handel (28\%) und Baugewerbe (35\%), am häufigsten im Bereich Banken/Versicherungen (68\%). Große Heterogenität besteht innerhalb des Wirtschaftsbereichs Landwirtschaft/Bergbau/Energie/Wasser: Lediglich $23 \%$ der Erwerbstätigen in der Land- und Forstwirtschaft teilten mit, dass BGF im Betrieb durchgeführt wurde, während im Bergbau/Energie/Wasser der analoge Anteil mit 74\% am höchsten von allen Wirtschaftszweigen ist. Den niedrigsten Verbreitungsgrad der BGF weist dagegen das Gastgewerbe auf. Nur 19\% der Erwerbstätigen im Gastgewerbe bekunden ein BGF-Angebot im Betrieb.

Ein kleiner Teil der Erwerbstätigen ist bei einer Leiharbeitsfirma angestellt, die sie an andere Unternehmen vermittelt. Von den Leiharbeitnehmern teilen lediglich $32 \%$ mit, dass in ihrem Entleih-Betrieb BGF angeboten wurde.

Die Verbreitung und Inanspruchnahme von BGF korreliert mit Befristung des Arbeitsverhältnisses. Arbeiter und Angestellte in einem befristeten Arbeitsverhältnis berichten in wesentlich geringerem Ausmaß als die unbefristeten Erwerbstätigen ein BGF-Angebot im Betrieb (39 vs. $49 \%$ ) und nehmen signifikant weniger daran teil (47 vs. $60 \%$ ).

- Tab. 1 informiert auch über enge Assoziationen zwischen drohender Entlassung und BGF. Je höher die selbst eingeschätzte Entlassungsgefahr, desto weniger Erwerbstätige nennen BGF-Angebote und umso niedriger sind ihre Inanspruchnahmequoten.

Die $>$ Abb. 2 präsentiert den Anteil der Erwerbstätigen, in deren Betrieb Gesundheitsförderung in den letzten 2 Jahren umgesetzt wurde, nach der Klassifikation der European Socioeconomic Groups (ESeG). Das Balkendiagramm offenbart bemerkenswerte Unter-

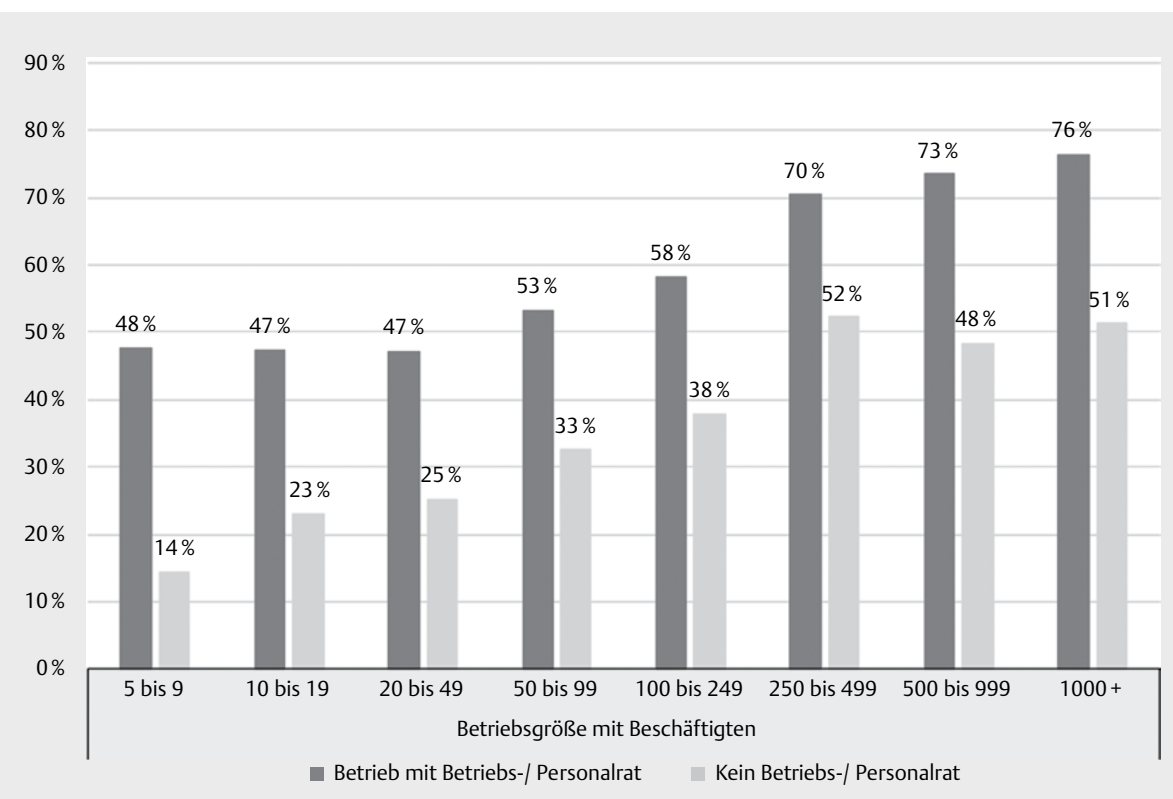

Abb. 1 Anteil der Erwerbstätigen mit Betrieblichem Gesundheitsförderungsangebot nach Betriebsgröße und Betriebs- bzw. Personalrat in der BIBB-/BAuA-Erwerbstätigenbefragung 2018. 1) $\mathrm{N}=16008$. 2) Betriebe mit mindestens 5 Beschäftigten. 3) Chi-Quadrat = 2322,69; $d f=7 ; p<0,001$. 


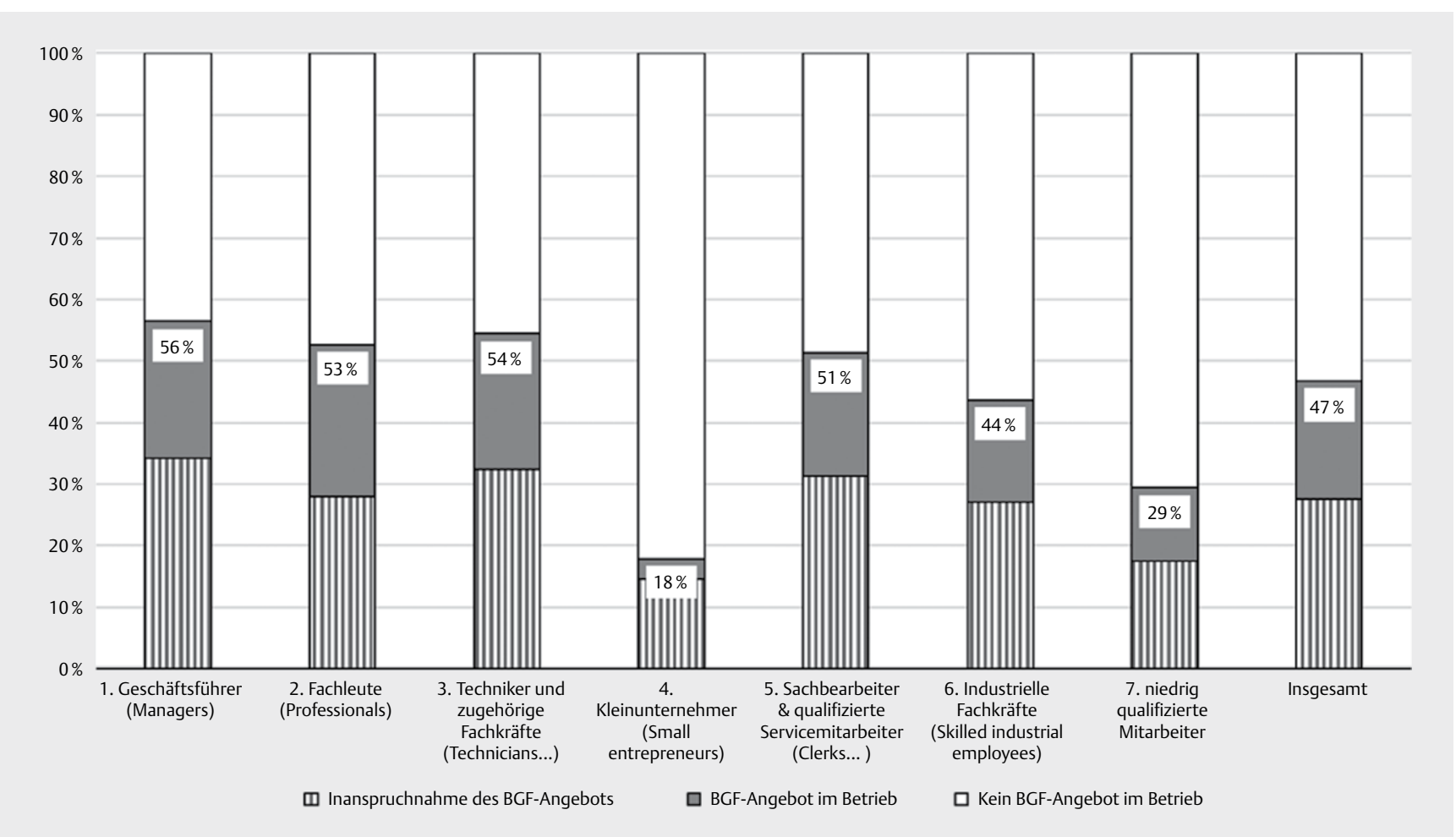

Abb. 2 Anteil der Erwerbstätigen mit Betrieblichem Gesundheitsförderungsangebot nach sozio-ökonomischem Status (European Socioeconomic Groups (ESeG)) in der BIBB-/BAuA-Erwerbstätigenbefragung 2018. Anmerkungen: 1) Die Gruppen unterscheiden sich beim BGF-Angebot (Chi-Qua$\mathrm{drat}=733,84 ; \mathrm{df}=6 ; \mathrm{p}<0,001$ ) und bei der Inanspruchnahme (Chi-Quadrat = 55,67; $\mathrm{df}=6 ; \mathrm{p}<0,001$ ) in statistisch signifikanter Weise. 2 ) $\mathrm{N}=18022$. 3) Die Gruppe der Kleinunternehmer enthält keine Solo-Selbstständigen und Ein-Personen-Betriebe, da denen die Fragen zur BGF erhebungstechnisch nicht gestellt wurden. 4) Originalbezeichnung der ESeG [37] in Klammern.

schiede zwischen den 7 ESeG-Hauptgruppen bei der BGF. Es belegt, dass den Managern am häufigsten betriebliche Gesundheitsförderung angeboten wurde. Von ihnen sagt über die Hälfte (56\%) aus, dass es ein BGF-Angebot gibt, was von $61 \%$ angenommen wurde. Es folgen mit geringem Abstand die Techniker und zugehörigen Fachkräfte, die Fachleute („Professionals“) sowie Sachbearbeiter und qualifizierten Servicemitarbeiter. Am seltensten wird dagegen eine Durchführung der BGF von Kleinunternehmern genannt (18\%). Weit unterdurchschnittlich ist auch der Anteil von 29\% unter den niedrig qualifizierten Mitarbeitern, die BGF-Angebote im Betrieb berichten.

BGF geht mit steigender Arbeitszufriedenheit einher ( $\triangleright$ Tab. 1). Von den Erwerbstätigen, die mit ihrer Arbeit alles in allem sehr zufrieden waren, geben $54 \%$ ein BGF-Angebot im Betrieb und eine hohe Inanspruchnahmequote von $63 \%$ an. Unter den unzufriedenen Erwerbstätigen ist sowohl der Anteil von $20 \%$, der BGF-Angebote berichtet, als auch die Inanspruchnahmequote mit $38 \%$ außerordentlich niedrig.

Wie Abb. 3 demonstriert, besteht dieser Zusammenhang zwischen BGF-Angebot und Arbeitszufriedenheit nicht nur allgemein, sondern in einem breiten Spektrum von Einzelaspekten. Es zeigt sich durchgängig, dass der Anteil der Erwerbstätigen, die BGF-Angebote im Betrieb nennen, mit dem Zufriedenheitsgrad signifikant ansteigt.

Die binäre logistische Regressionsanalyse in $>$ Tab. 2 zielt darauf, ob BGF nach Aussagen der Erwerbstätigen in ihrem Betrieb an- geboten wurde. Für die Vorhersage der Wahrscheinlichkeit werden als Prädiktoren die sozio-demografischen Merkmale, die subjektive Gesundheit, Merkmale des Betriebs und die Arbeitszufriedenheit einbezogen.

In der multivariaten Betrachtungsweise haben Frauen eine signifikant geringere Chance als Männer $(\mathrm{OR}=0,83)$, dass in ihrem Betrieb BGF angeboten wird.

Ebenso ist die Wahrscheinlichkeit eines BGF-Angebots für Erwerbstätige mit ausländischer Staatsangehörigkeit im Vergleich zu deutschen Erwerbstätigen signifikant reduziert $(O R=0,78)$.

Im Vergleich zu Erwerbstätigen ohne Berufsabschluss verbessert ein höherer Ausbildungsabschluss signifikant die Wahrscheinlichkeit eines BGF-Angebots, vor allem nach einer Aufstiegsfortbildung wie beim Meister $(O R=1,61)$ oder bei Hochschulabschluss bzw. für gehobene bzw. höhere Beamte $(O R=1,50)$.

Als stärkste Einflussvariable wurde in der multivariaten Analyse die Betriebsgröße identifiziert, die in diesem Modell in drei Kategorien untersucht wurde. Im Verhältnis zu den Kleinbetrieben bis 49 Beschäftigten ist in mittleren Unternehmen mit 50 bis 249 Beschäftigten die Wahrscheinlichkeit für ein BGF-Angebot mehr als doppelt und in großen Unternehmen mit mindestens 250 Beschäftigten mehr als fünffach gesteigert $(O R=2,17$ und $O R=5,64)$.

Das BGF-Angebot variiert signifikant nach den Wirtschaftsbereichen des Betriebs. Im Vergleich zum Öffentlichen Dienst sind die Chancen auf ein entsprechendes Angebot in Industrie, Handwerk, Handel, sonstigen Dienstleistungen und anderen Wirtschaftsbe- 


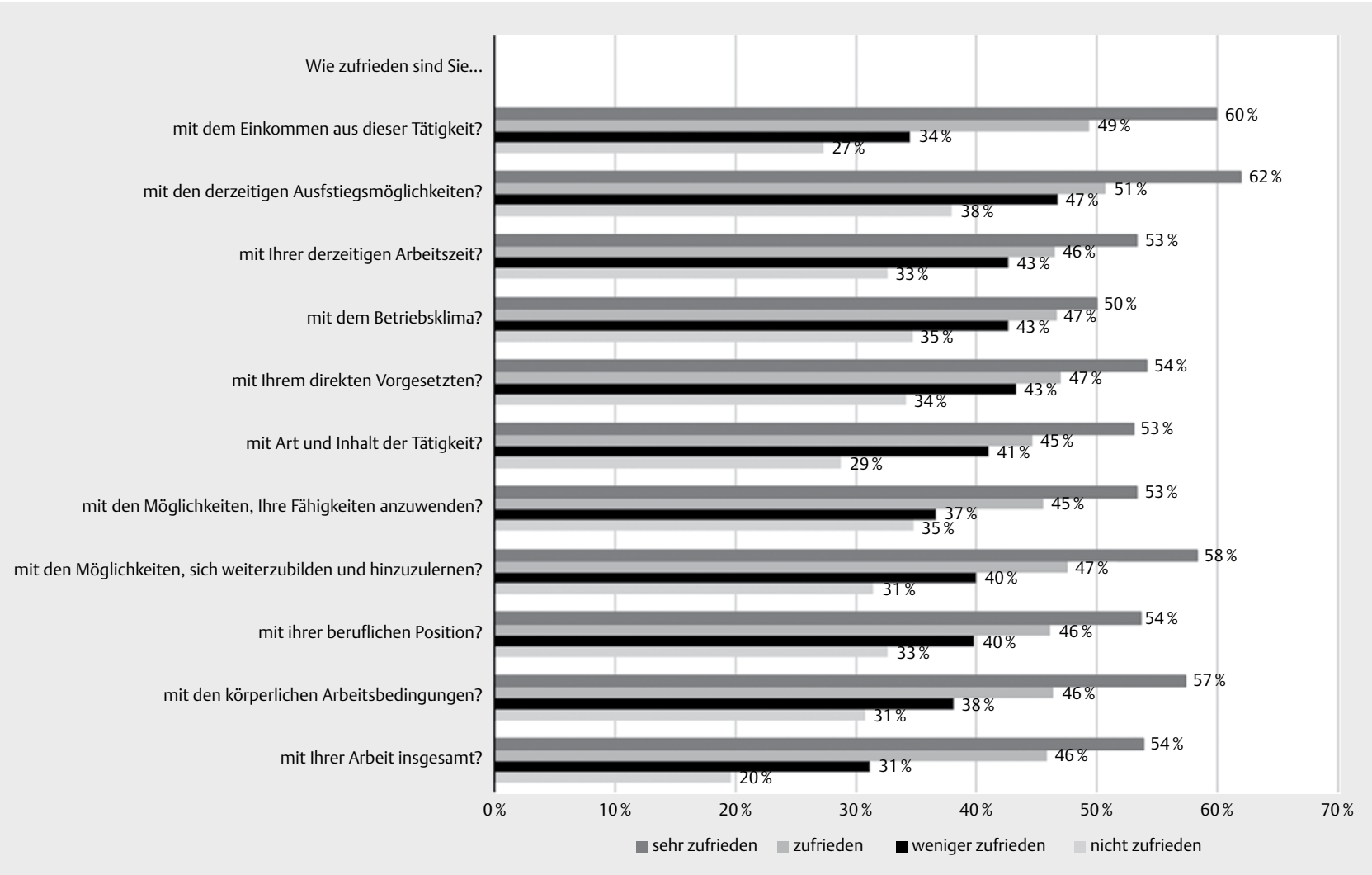

- Abb. 3 Anteil der Erwerbstätigen mit Betrieblichem Gesundheitsförderungsangebot nach Arbeitszufriedenheit in der BIBB-/BAuA-Erwerbstätigenbefragung 2018. Anmerkung: Die 4 Gruppen zur Zufriedenheit unterscheiden sich im BGF-Angebot bei allen Merkmalen der Abbildung in statistisch signifikanter Weise $(p<0,001)$.

reichen stark verringert. Das trifft vor allem auf Handwerk und Handel zu $(O R=0,36$ und $O R=0,37)$.

Ein sehr guter oder ausgezeichneter Gesundheitszustand geht mit einer etwas gesteigerten Odds Ratio von 1,15 für BGF im Betrieb einher.

In Betrieben, in denen die Erwerbstätigen mit der Arbeit insgesamt sehr zufrieden sind, ist die Wahrscheinlichkeit eines BGF-Angebots statistisch signifikant um 1,70-fach erhöht.

\section{Diskussion}

Die BIBB/BAuA-Erwerbstätigenbefragung 2018 gibt Aufschluss über die BGF in Deutschland, die bei fast der Hälfte (47\%) der Erwerbstätigen im Betrieb umgesetzt wird. Die Ergebnisse deuten auf einen moderaten Ausbau der BGF in Deutschland im Abgleich zur Erhebungswelle 2011/12 hin, in der der entsprechende Anteil bei $44 \%$ lag [6]. Gleichzeitig setzt sich mit einer Teilnahme von nur $59 \%$ ein rückläufiger Trend in 2018 fort. Die Inanspruchnahmequoten betrugen in den Erhebungswellen 2011/12 und 2005/06 noch 62 und $65 \%$ [6]. Damit relativiert sich das Wachstum in der BGF und es sind nur noch kleine Fortschritte im Verbreitungsgrad zu konstatieren - trotz Präventionsgesetz 2015 und nationaler Präventionsstrategie. Die in der GKV-Präventionsberichterstattung [20] parallel propagierten hohen Steigerungsraten der erreichten Betriebe und Beschäftigten bilden sich in dieser Wiederholungsbefragung wenig ab.
Ein starker Einflussfaktor für die Durchführung von BGF in Deutschland ist nach wie vor die Betriebsgröße, was sich schon in früheren Erhebungswellen [6] und vielen Studien gezeigt hat $[3,4]$. Die Auswertung verifiziert die sehr geringe Verbreitung der BGF in den Kleinst- und Kleinbetrieben. Mit der Betriebsgröße nimmt die Durchführung von BGF stark zu, während parallel die Inanspruchnahmequoten abnehmen. BGF kann in großen Betrieben auf den vorhandenen Strukturen zum Gesundheitsmanagement aufsetzen. Möglicherweise sind in größeren Betrieben jedoch die Kapazitäten in der BGF nicht ausreichend für die Beschäftigtenzahl dimensioniert oder die Maßnahmen tendenziell weniger passgenau auf die Bedürfnisse der Belegschaft hin konzipiert. Die Analysen zeigen auch die von der Betriebsgröße unabhängigen Einflussnahmen des Wirtschaftsbereichs und der Stellung im Beruf auf die Umsetzung der BGF, die in Übereinstimmung mit der Forschung stehen $[3,4,6,8,10]$.

Die Auswertungen bestätigen den Zusammenhang zwischen BGF und Betriebs-/Personalrat, der die Implementierung und Aufrechterhaltung der BGF unterstützen kann. Eine Korrelation wurde auch in anderen Studien festgestellt $[4,8]$.

Die Analysen offenbaren, dass Erwerbstätige mit niedrigem sozioökonomischen Status verhältnismäßig wenig von der BGF erreicht werden. Relativ gering ist die Realisierung der BGF in Betrieben von Erwerbstätigen, die keinen Berufsabschluss haben, in einem befristeten Arbeitsverhältnis stehen, von Entlassung bedroht sind, keine deutsche Staatsangehörigkeit besitzen oder als Leiharbeitnehmer 
> Tab. 2 Angebot Betrieblicher Gesundheitsförderung in der BIBB-/BAuA-Erwerbstätigenbefragung 2018. Ergebnisse binärer logistischer Regression.

\begin{tabular}{|c|c|c|}
\hline Merkmale & & Odds Ratio (95\%-KI) \\
\hline \multicolumn{3}{|l|}{ Soziodemografie } \\
\hline \multirow[t]{2}{*}{ Geschlecht } & Männer & Ref. \\
\hline & Frauen & $0,83(0,77-0,89)^{* * *}$ \\
\hline \multirow[t]{4}{*}{ Höchster Ausbildungsabschluss } & Ohne Berufsabschluss & Ref. \\
\hline & Duale oder schulische Berufsausbildung, einfache oder mittlere Beamte & $1,29(1,13-1,47)^{* * *}$ \\
\hline & Aufstiegsfortbildung (Meister, Techniker u.ä.) & $1,61(1,35-1,92)^{* * *}$ \\
\hline & Fachhochschule, Universität, BA, gehobene, höhere Beamte & $1,50(1,30-1,73)^{* * *}$ \\
\hline \multirow[t]{4}{*}{ Stellung im Beruf } & Arbeiter & Ref. \\
\hline & Angestellter & $1,28(1,16-1,41)^{* * *}$ \\
\hline & Beamter & $1,17(0,98-1,41)$ \\
\hline & Sonstige & $0,80(0,66-0,96)^{*}$ \\
\hline \multirow[t]{2}{*}{ Staatsangehörigkeit } & Deutsch & Ref. \\
\hline & Andere Staaten & $0,78(0,69-0,87)^{* * *}$ \\
\hline \multicolumn{3}{|l|}{ Betriebsmerkmale } \\
\hline \multirow[t]{3}{*}{ Betriebsgröße } & bis 49 Beschäftigte & Ref. \\
\hline & 50 bis 249 Beschäftigte & $2,17(1,99-2,36)^{* * *}$ \\
\hline & $250+$ Beschäftigte & $5,64(5,16-6,16)^{* * *}$ \\
\hline \multirow[t]{6}{*}{ Wirtschaftsbereich } & Öffentlicher Dienst & Ref. \\
\hline & Industrie & $0,73(0,66-0,82)^{* * *}$ \\
\hline & Handwerk & $0,36(0,31-0,41)^{* * *}$ \\
\hline & Handel & $0,37(0,32-0,43)^{* * *}$ \\
\hline & sonstige Dienstleistungen & $0,45(0,41-0,50)^{* * *}$ \\
\hline & andere Wirtschaftsbereiche & $0,54(0,48-0,62)^{* * *}$ \\
\hline \multicolumn{3}{|l|}{ Gesundheit } \\
\hline \multirow[t]{2}{*}{ Subjektiver Gesundheitszustand } & gut, weniger gut oder schlecht & Ref. \\
\hline & ausgezeichnet oder sehr gut & $1,15(1,06-1,24)^{* * *}$ \\
\hline \multicolumn{3}{|l|}{ Arbeitsplatzbezogene Merkmale } \\
\hline \multirow[t]{2}{*}{ Arbeitszufriedenheit } & zufrieden, weniger oder nicht zufrieden & Ref. \\
\hline & sehr zufrieden & $1,70(1,58-1,84)^{* * *}$ \\
\hline Pseudo-R2 (Nagelkerke) & 0,256 & \\
\hline -2 Log-Likelihood & 19741,239 & \\
\hline$(\mathrm{N})$ & $(17.313)$ & \\
\hline
\end{tabular}

beschäftigt sind. Die GKV hat sich das Ziel gesetzt, Zahl und Anteil der Betriebe mit einem hohen Anteil Beschäftigter ohne abgeschlossene Berufsausbildung in der BGF zu erhöhen [20, 38]. Um zur Verminderung sozial bedingter Ungleichheit von Gesundheitschancen beizutragen, braucht es in der BGF daher proaktive zielgruppenspezifische Ansprachestrategien und ein möglichst flächendeckendes BGF-Angebot.

Ein überraschender Befund ist, dass von den Erwerbstätigen, die ihren Gesundheitszustand als schlecht bewerten, lediglich ein Drittel die Durchführung von BGF im Betrieb angeben. Es wäre zu erwarten gewesen, dass Arbeitgeber oder auch Sozialversicherungen nach Bedarfsanalysen besonders bei Erwerbstätigen mit schlechterer Gesundheit ansetzen würden. Der GKV-Leitfaden Prävention [38] schreibt eine Ermittlung des Handlungsbedarfs für den Betrieb als Ganzes sowie der einzelnen Beschäftigtengruppen vor. Hier besteht weiterer Forschungsbedarf.

Die Arbeitszufriedenheit ist ein Schlüsselindikator für viele Bedingungen im Betrieb und am Arbeitsplatz. In der multivariaten
Betrachtung haben die Erwerbstätigen, die mit ihrer Arbeit insgesamt sehr zufrieden sind, eine stark erhöhte Chance auf ein BGFAngebot im Betrieb. Die BGF korreliert nicht nur mit höherer Arbeitszufriedenheit allgemein, sondern bei allen abgefragten Einzelaspekten. Ebenso nehmen die Inanspruchnahmequoten der BGF mit der allgemeinen Arbeitszufriedenheit erheblich zu. Das spricht für die BGF als integraler Bestandteil der Unternehmenskultur.

Die Ergebnisse legen außerdem nahe, dass Männer über den $\mathrm{Ar}$ beitsplatz besser von der Gesundheitsförderung angesprochen werden können als bei der individuellen verhaltensbezogenen Prävention im GKV-Kurssystem, an der sie sich kaum beteiligen [20].

Weiterer Forschungsbedarf besteht nicht nur bei der Verbreitung der BGF, sondern auch bei Art und Inhalt, Qualität und Ergebnissen der Maßnahmen. Für die bessere Erforschung der Nichtinanspruchnahme sollten zukünftig die Gründe erfasst werden. Die Diskrepanz zwischen BGF-Angebot und Inanspruchnahme ist noch ungeklärt und die Teilnehmerselektivität könnte auch auf die Passgenauigkeit der BGF-Maßnahmen zurückzuführen sein. Am nied- 
rigsten ist die Teilnahmequote bei den Erwerbstätigen, die nicht zufrieden mit ihrer Arbeit insgesamt sind.

Ein CATI-Survey bei Erwerbstätigen hat prinzipiell methodische Limitationen. Über die Selbstauskünfte der Interviewpersonen können lediglich deren Wahrnehmung und Kenntnisse eruiert werden. Die Antworten sind vom Erinnerungsvermögen wie bei der BGF in den letzten zwei Jahren abhängig. Antwortrichtungen im Sinne der sozialen Erwünschtheit können nicht ausgeschlossen werden. Gesundheitsbewusste Erwerbstätige informieren sich möglicherweise auch besser über ein BGF-Angebot im Betrieb. Ausländische Erwerbstätige wurden bei der Untersuchung nur einbezogen, wenn sie ausreichend Deutsch sprachen. Die Fallzahlen begrenzen statistisch die Auswertungstiefe. Einer etwaigen Verzerrung durch Unit Nonresponse wurde durch mehrere Maßnahmen in der Erhebungsmethode begegnet, darunter mit einem Mobilfunkanteil von $30 \%$ [34].

\section{FAZIT}

Die Implementierung der BGF ist freiwillig im Sinne der WHO-Charta der Gesundheitsförderung [1]. Knapp die Hälfte der Erwerbstätigen berichtet in der BIBB-/BAuAErwerbstätigenbefragung 2018 die Durchführung von BGF im Betrieb, die dann mehrheitlich angenommen wurde. Rund ein Viertel der Erwerbstätigen hat demnach an Maßnahmen der BGF in den letzten 2 Jahren teilgenommen. Derzeit ist nur eine Minderheit der Betriebe in der Gesundheitsförderung engagiert. Die Chance auf ein BGF-Angebot ist mit Betriebsmerkmalen, sozio-demografischen Charakteristika und beruflicher Qualifikation verbunden. Die Analysen ergeben enge Assoziationen mit der Arbeitszufriedenheit, dem subjektiven Gesundheitszustand und dem sozio-ökonomischem Status. Beim Umsetzungsgrad der BGF gibt es nur relativ kleine Fortschritte seit der letzten Erhebungswelle 2011/12. Im Sinne der „Gesundheit für Alle“-Strategie [1] braucht es größere Anreiz- und Unterstützungssysteme für kleine und mittlere Betriebe bei der BGF. Innerhalb der Betriebe erscheinen eine Adressierung von Erwerbstätigen mit niedrigem sozio-ökonomischem Status und eine Zielgruppenspezifizierung der BGF-Maßnahmen als notwendig.

\section{Interessenkonflikt}

Die Autorinnen/Autoren geben an, dass kein Interessenkonflikt besteht

\section{Literatur}

[1] Weltgesundheitsorganisation (WHO). Ottawa-Charta zur Gesundheitsförderung. Genf: 1986. Im Internet: https://www.euro.who.int/_ data/assets/pdf_file/0006/129534/Ottawa_Charter_G.pdf; Stand: 19.10.2021
[2] Europäisches Netzwerk für Betriebliche Gesundheitsförderung. Die Luxemburger Deklaration zur Betrieblichen Gesundheitsförderung. Berlin: 2014. Im Internet: https://www.dnbgf.de/fileadmin/ downloads/materialien/dateien/2014_Luxemburger_Deklaration_BGF. pdf; Stand: 19.10.2021

[3] Faller G. Umsetzung Betrieblicher Gesundheitsförderung/Betrieblichen Gesundheitsmanagements in Deutschland. Stand und Entwicklungsbedarfe der einschlägigen Forschung. Gesundheitswesen 2018; 80: 278-285 . doi: 10.1055/s-0042-100624

[4] Hollederer A, Wießner F. Prevalence and development of workplace health promotion in Germany: results of the IAB Establishment Panel 2012. International archives of occupational and environmental health 2015; 88: 861-873 . doi: 10.1007/s00420-014-1012-z

[5] Hollederer A. Betriebliche Gesundheitsförderung in Deutschland. Ergebnisse des IAB-Betriebspanels 2002 und 2004. Gesundheitswesen 2007; 69: 63-76 . doi: 10.1055/s-2007-970599

[6] Beck D, Lenhardt U. Betriebliche Gesundheitsförderung in Deutschland: Verbreitung und Inanspruchnahme. Ergebnisse der BIBB/ BAuA-Erwerbstätigenbefragungen 2006 und 2012. Gesundheitswesen 2016; 78: 56-62 . doi: 10.1055/s-0034-1387744

[7] Slesina W. Betriebliche Gesundheitsförderung in der Bundesrepublik Deutschland. Bundesgesundheitsblatt, Gesundheitsforschung, Gesundheitsschutz 2008; 51: 296-304

[8] Beck D, Lenhardt U, Schmitt B et al. Patterns and predictors of workplace health promotion. cross-sectional findings from a company survey in Germany. BMC public health 2015; 15: 343-351

[9] Institut DGB-Index Gute Arbeit (Hg.). DGB-Index Gute Arbeit. Der Report 2013. Berlin; 2014

[10] Hessenmöller A-M, Pangert B, Pieper C et al. iga.Barometer 4. Welle 2013: Die Arbeitssituation in Unternehmen. 1. Aufl. 2014

[11] Bechmann S, Jäckle R, Lück $P$ et al. Motive und Hemmnisse für Betriebliches Gesundheitsmanagement (BGM). iga.Report 20. 2. Aufl. Berlin: 2011

[12] Hollederer A. Betriebliche Gesundheitsförderung bei älteren Beschäftigten in Deutschland. Ergebnisse im IAB-Betriebspanel 2011. Zeitschrift für Gerontologie und Geriatrie 2016; 49: 132-137

[13] Huber M, Lechner M, Wunsch C. Workplace Health Promotion and Labour Market Performance of Employees. IZA-Disscussion Paper. Bonn: 2014

[14] Schaefer E, Drexler H, Kiesel ]. Betriebliche Gesundheitsförderung in kleinen, mittleren und großen Unternehmen des Gesundheitssektors. Gesundheitswesen 2016; 78: 161-165. doi: 10.1055/s-0034-1396887

[15] Ansmann L, Jung J, Nitzsche A et al. Zusammenhänge zwischen der Betriebsstruktur und Betrieblichem Gesundheitsmanagement in der Informationstechnologie- und Kommunikationsbranche. Gesundheitswesen 2012; 74: 298-305

[16] Jung J, Nitzsche A, Ansmann L et al. Organizational factors and the attitude toward health promotion in German ICT-companies. Health promotion international 2012; 27: 382-393

[17] Zelfel RC, Alles T, Weber A. Gesundheitsmanagement in kleinen und mittleren Unternehmen - Ergebnisse einer repräsentativen Unternehmensbefragung. Gesundheitswesen 2011; 73: 515-519

[18] Gröben F, Wenninger S. Betriebliche Gesundheitsförderung im öffentlichen Dienst. Präv Gesundheitsf 2006; 1: 94-98

[19] Plath S-C, Köhler T, Krause $\mathrm{H}$ et al. Prevention, health promotion and workplace health management in German banks: Results from a nationwide representative survey. J Public Health 2008; 16: 195-203

[20] Medizinischer Dienst des Spitzenverbandes Bund der Krankenkassen e. V. (MDS), GKV-Spitzenverband. Präventionsbericht 2020. Essen, Berlin; 2020. Im Internet: https://www.gkv-spitzenverband.de/media/ dokumente/krankenversicherung_1/praevention__selbsthilfe__beratung/praevention/praeventionsbericht/2020_GKV_MDS_Praeventionsbericht.pdf; Stand: 19.10.2021 
[21] Nationale Präventionskonferenz. Erster Präventionsbericht nach § 20d Abs. 4 SGB V. Berlin: 2019. Im Internet: https://www.npk-info. de/fileadmin/user_upload/ueber_die_npk/downloads/2_praeventionsbericht/NPK-Praeventionsbericht_Barrierefrei.pdf; Stand: 19.10.2021

[22] Siegrist J, Rosskam E, Leka S. Work and worklessness. Final report of the Task group on employment and working conditions, including occupation, unemployment and migrant workers. Kopenhagen: 2016

[23] Dragano N, Wahrendorf M, Müller K et al. Arbeit und gesundheitliche Ungleichheit: Die ungleiche Verteilung von Arbeitsbelastungen in Deutschland und Europa. Bundesgesundheitsbl 2016; 59: 217-227

[24] Kroll LE, Müters S, Schumann M et al. Wahrnehmung gesundheitsgefährdender Arbeitsbedingungen in Deutschland. Robert Koch-Institut (RKI) Journal of Health Monitoring 2017; 124-129

[25] BKK Dachverband. BKK Gesundheitsreport 2020. Mobilität - Arbeit Gesundheit. Berlin: MWV Medizinisch Wissenschaftliche Verlagsgesellschaft. 2020. Im Internet: https://www.bkk-dachverband.de/ publikationen/bkk-gesundheitsreport; Stand: 19.10.2021

[26] Deutscher Bundestag. Unterrichtung durch die Bundesregierung: Erster Bericht der Nationalen Präventionskonferenz über die Entwicklung der Gesundheitsförderung und Prävention (Erster Präventionsbericht) mit Stellungnahme der Bundesregierung. Drucksache 19/26140. Berlin: 2021

[27] Kuoppala J, Lamminpää A, Husman P. Work health promotion, job well-being, and sickness absences. A systematic review and metaanalysis. Journal of occupational and environmental medicine 2008; 50: $1216-1227$

[28] Barthelmes I, Bödeker W, Sörensen J. Wirksamkeit und Nutzen arbeitsweltbezogener Gesundheitsförderung und Prävention. Zusammenstellung der wissenschaftlichen Evidenz 2012 bis 2018. iga. Report 40. Dresden: 2019

[29] Rongen A, Robroek SJW, van Lenthe F] et al. Workplace health promotion: a meta-analysis of effectiveness. American journal of preventive medicine 2013; 44: 406-415. doi: 10.1016/j.amepre.2012.12.007
[30] Tarro L, Llauradó E, Ulldemolins G et al. Effectiveness of Workplace Interventions for Improving Absenteeism, Productivity, and Work Ability of Employees. A Systematic Review and Meta-Analysis of Randomized Controlled Trials. International journal of environmental research and public health 2020; 17. doi: 10.3390/ijerph17061901

[31] Basińska-Zych A, Springer A. Organizational and Individual Outcomes of Health Promotion Strategies. A Review of Empirical Research. International journal of environmental research and public health 2021; 18. doi: 10.3390/ijerph18020383

[32] Baxter S, Sanderson K, Venn AJ et al. The relationship between return on investment and quality of study methodology in workplace health promotion programs. American journal of health promotion: AJHP 2014; 28: 347-363

[33] Lück M, Hünefeld L, Brenscheidt S et al. Grundauswertung der BIBB/ BAuA-Erwerbstätigenbefragung 2018. 2. Aufl. Dortmund: Bundesanstalt für Arbeitsschutz und Arbeitsmedizin (BAuA); 2019. doi:10.21934/baua:bericht20190618

[34] Rohrbach-Schmidt D, Hall A. BIBB/BAuA Employment Survey 2018. BIBB-FDZ Data and Methodological Report 1/2020. Bonn: 2020. Zugriff unter https://www.bibb.de/veroeffentlichungen/en/publication/show/16563

[35] Statistisches Bundesamt. Klassifikation der Wirtschaftszweige 2008 (WZ 2008). Wiesbaden, Germany; 2008

[36] Bohr J. EU-AES Tools: Implementation of the European Socioeconomic Groups Classification (ESeG) using Adult Education Survey Microdata: SSOAR - GESIS Leibniz Institute for the Social Sciences; 2018

[37] Meron M, Amar M, Laurent-Zuani A-C et al. Final Report of the ESSnet on the harmonisation and implementation of a European socio-economic classification. European Socio-economic Groups (ESeG). Luxembourg: 2014

[38] GKV-Spitzenverband. Leitfaden Prävention. Berlin: 2020. Im Internet: https://www.gkv-spitzenverband.de/media/dokumente/krankenversicherung_1/praevention__selbsthilfe__beratung/praevention/ praevention_leitfaden/Leitfaden_Pravention_2020_barrierefrei.pdf; Stand: 19.10 .2021 